\title{
Search for scalar top and bottom quarks at the Tevatron
}

\author{
Philippe Calfayan, on behalf of the D0 and CDF collaborations \\ Department für Physik, Ludwig-Maximilians-Universität München, Am Coulombwall 1, D-85748 Garching, \\ Germany
}

\begin{abstract}
This document reviews recent results on the search for scalar top and scalar bottom quarks in $p \bar{p}$ collisions at $\sqrt{s}=1.96 \mathrm{TeV}$. The analyses presented are based on data samples with integrated luminosities from 1.0 to $1.9 \mathrm{fb}^{-1} \mathrm{recorded}$ at the Tevatron with the D0 and CDF detectors.
\end{abstract}

Keywords: Supersymmetry, Tevatron, D0, CDF, scalar top quark, scalar bottom quark

PACS: $12.60 .-1,12.60 . J v, 14.80 . \mathrm{Ly}$

\section{INTRODUCTION}

Supersymmetry (SUSY) [1] provides the unification of matter and interactions. A SUSY operator acts on the spin of a particle and allows the interchange of fermions and bosons. The new particles arising from this symmetry are referred to as the superpartners. Since no SUSY particle has yet been observed, SUSY must be broken. The simplest SUSY extension of the Standard Model (SM), referred to as the Minimal Supersymmetric Standard Model (MSSM), introduces a minimal addition of superpartners and includes a Higgs sector consisting of two doublets.

The stability of the proton in the MSSM can be ensured by the conservation of a new discrete and multiplicative quantum number, namely the R-parity $(R)$, which is defined by $R=(-1)^{3 B+2 S+L}$, where $S$ is the spin of the particle, and $B$ and $L$ the baryon and lepton numbers, respectively. $R$ is equal to 1 and -1 for SM and SUSY particles, respectively. Assuming $R$ is conserved, SUSY particles would therefore only be produced in pairs, and would only decay to another SUSY particle in association with a SM particle. The lightest SUSY particle (LSP) is thus stable and constitutes a serious candidate for dark matter if it is colorless and neutral. In the analyses presented in this note, the MSSM is the underlying SUSY model, and $R$ is conserved.

The superpartner of the top and the bottom quarks are the scalar top (stop, $\tilde{t}$ ) and the scalar bottom (sbottom, $\tilde{b}$ ) quarks, respectively. The superpartners of both helicity states of the SM quarks are supposed to be mass degenerate for the two first generations, while for the third one, the high mass of the top quark $\left(m_{t}\right)$ and a large value for $\tan (\beta)$ (the ratio of the vacuum expectation values of the two Higgs doubles) imply a significant mass splitting in the $\tilde{t}$ and $\tilde{b}$ sectors, respectively. The lightest $\tilde{t}$ and $\tilde{b}$ could therefore be within the reach of the Tevatron, and would predominantly be produced via the strong coupling.

\section{PAIR PRODUCTION OF SCALAR BOTTOM QUARKS VIA GLUINOS}

The CDF search for gluino-mediated $\tilde{b}$ pair production is performed in the final state consisting of four $b$-jets and missing transverse energy $\left(Z_{T}\right)$ [2]. The analysis is based on a data set with a luminosity of $1.8 \mathrm{fb}^{-1}$. The gluinos ( $\tilde{g}$, the superpartners of the SM gluons) are assumed to be produced in pairs and to decay exclusively to a $\tilde{b}$ and a $b$-quark. The lightest neutralino, $\tilde{\chi}_{1}^{0}$, which results from the mixing of the superpartners of the SM neutral gauge and Higgs bosons, is considered to be the LSP. In this study, both $\tilde{b}$ decay with a branching fraction of $100 \%$ to a $\tilde{\chi}_{1}^{0}$ and a $b$-quark through a two-body decay.

The main backgrounds are $t \bar{t}$ and QCD multijet production. At least one $b$-tagged jet is required and events with $\not_{T}$ lower than $70 \mathrm{GeV}$ are rejected. The signal enhancement is optimized in two regions. One of them is defined by $m_{\tilde{g}}=320 \mathrm{GeV}$ and $m_{\tilde{b}}=250 \mathrm{GeV}$, and the other one by $m_{\tilde{g}}=300 \mathrm{GeV}$ and $m_{\tilde{b}}=280 \mathrm{GeV}$.

Upper limits on the $\tilde{b}$ pair production cross section are computed and found not to exceed $0.1 \mathrm{pb}$. All cross section limits reported in this note are defined at $95 \%$ confidence level. By comparing the upper cross section limits with the next-to-leading order (NLO) prediction, a mass exclusion region is derived as function of the $\tilde{b}$ and $\tilde{g}$ masses, as represented in Figure 1.

\section{DIRECT PAIR PRODUCTION OF SCALAR TOP QUARKS}

\subsection{The acoplanar $c$-jets $+Z_{T}$ final state}

The D0 collaboration published an analysis investigating the pair production of $\tilde{t}$ in the acoplanar final state composed of two $c$-jets and missing transverse energy [3]. $\tilde{\chi}_{1}^{0}$ is assumed to be the LSP, and the branching 


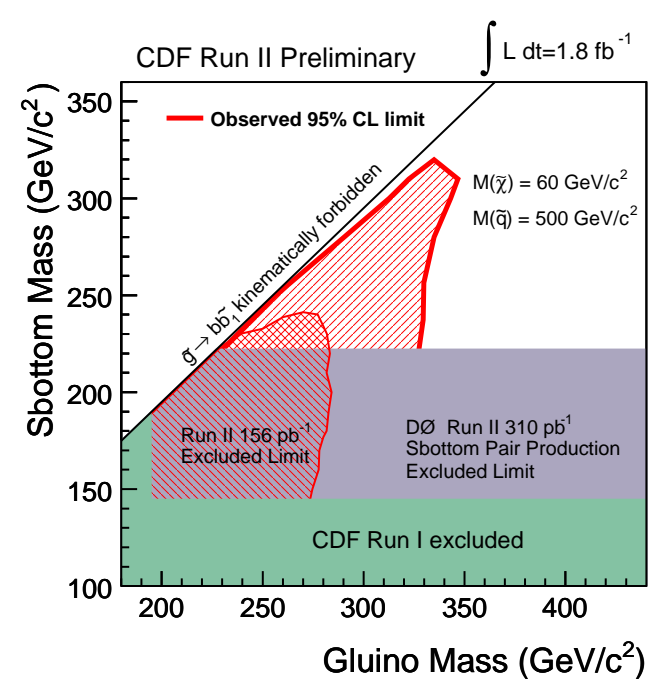

FIGURE 1. Search for $\tilde{g}$-mediated $\tilde{b}$ pair production in the $b$ jets $+Z_{T}$ final state. The mass exclusion region is parametrized as function of $m_{\tilde{b}}$ and $m_{\tilde{g}}$.

fraction for the loop-induced decay of the $\tilde{t}$ into a $c$-quark and a $\tilde{\chi}_{1}^{0}$ is set to $100 \%$. A data sample of $1 \mathrm{fb}^{-1}$ is utilized and was collected with triggers based on jets and $E_{T}$.

The main background sources are found to be $W$ and $Z$ boson production in association with jets. The instrumental background is removed by cutting on variables constructed from the azimuthal separation between the jets and $E_{T}$, and from the asymmetry between $E_{T}$ and the sum of all the jets transverse momenta $\left(H_{T}\right)$. At least one heavy-flavor jet is required to be tagged using a neural network tagger. Final selections consist of cuts on $\mathbb{E}_{T}, H_{T}$, and on the sum of the maximal and minimal azimuthal separation between the jets and $Z_{T}$. The optimization is achieved by maximizing the expected lower limit on $m_{\tilde{\chi}_{1}^{0}}$ for a given $m_{\tilde{t}}$.

Figure 2 provides the mass exclusion region parametrized as function of $m_{\tilde{\chi}_{1}^{0}}$ and $m_{\tilde{t}}$. The yellow band on the plot corresponds to the uncertainty on the signal cross section.

\subsection{The $b \bar{b} e^{ \pm} \mu^{ \pm} \tilde{v} \bar{v}$ final state}

The search for the pair production of $\tilde{t}$ followed by the three-body decays into a $b$-quark, a lepton, and a $\tilde{v}$ is performed by D0, using a data set of $1.1 \mathrm{fb}^{-1}$ [4]. The superpartner of the neutrino, $\tilde{v}$, is assumed to be the LSP. The branching fraction of the $\tilde{t}$ decay, which is realized through a virtual chargino, is considered to be $100 \%$. One of the two $\tilde{t}$ decays produces an electron, and the other

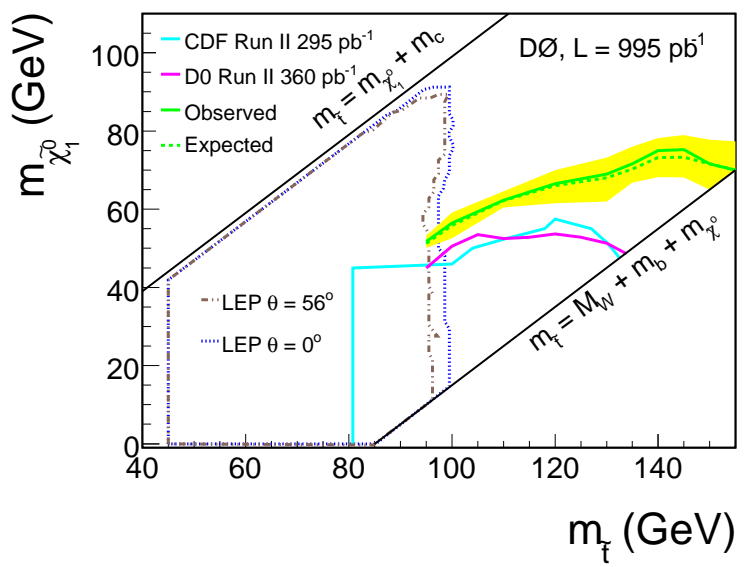

FIGURE 2. Search for $\tilde{t}$ pair production in the acoplanar $c$ jets $+E_{T}$ final state. The mass exclusion region is parametrized as function of $m_{\tilde{\chi}_{1}^{0}}$ and $m_{\tilde{t}}$.

one a muon. Events are selected with triggers firing on either electrons or muons with low transverse momenta.

The main backgrounds are $W W$ and $t \bar{t}$ production together with background of instrumental origin. No $b$ tagging is required. The selection cuts are based on the transverse mass constructed from the four vector of the lepton and $E_{T}$, and on the azimuthal separation between the lepton and $Z_{T}$.

Upper limits on the $\tilde{t}$ pair production cross section are calculated using $3 \times 4$ bins in the plane given by the scalar sum of $Z_{T}$ and the selected leptons transverse momenta, and by the sum of all the jets transverse momenta.

A mass exclusion region parametrized as function of $m_{\tilde{v}}$ and $m_{\tilde{t}}$ is obtained by interpreting the upper limits on the $\tilde{t}$ pair production cross section as limits on $m_{\tilde{t}}$ for a given $m_{\tilde{v}}$. The exclusion plot is shown in Figure 3.

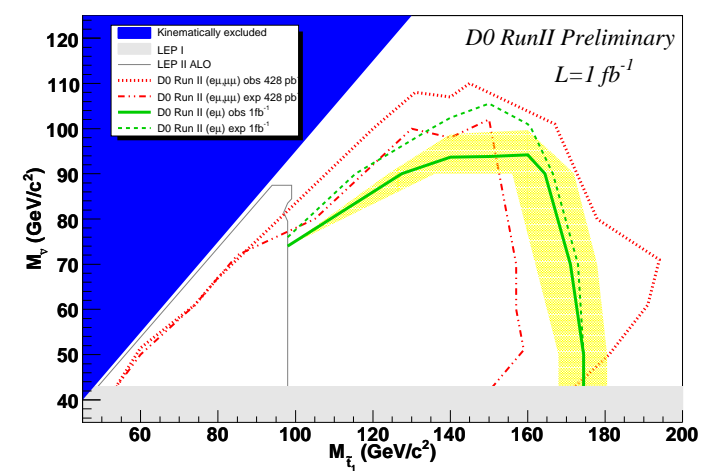

FIGURE 3. Search for $\tilde{t}$ pair production in the $b \bar{b} e^{ \pm} \mu^{ \pm} \tilde{v} \overline{\tilde{v}}$ final state. The mass exclusion region is parametrized as function of $m_{\tilde{v}}$ and $m_{\tilde{t}}$. 


\subsection{The $t \bar{t}$ lepton+jets final state}

The search for pair-produced $\tilde{t}$ is performed using the $t \bar{t}$ lepton plus jets signature, using nearly $1 \mathrm{fb}^{-1}$ of data collected with the D0 detector [5]. The LSP is considered to be the lightest neutralino, and $m_{\tilde{t}}$ is required not to exceed $m_{t}$. In the scenario investigated, the $\tilde{t}$ decays into a $b$-quark and the lightest chargino, $\tilde{\chi}_{1}^{ \pm}$, which results from the mixing of the superpartners of the SM charged gauge and Higgs bosons. $\tilde{\chi}_{1}^{ \pm}$is then assumed to decay into a $\tilde{\chi}_{1}^{0}$ and a $W$ boson. One of the $W$ boson decays into hadrons and the other one into leptons. The channels based on electron plus jets and muon plus jets are combined.

$W+$ jets and $t \bar{t}$ production are found to be the main contributions to the background. Only events with at least one $b$-tagged jet are selected.

The procedure to set upper limits on the $\tilde{t}$ pair production cross section relies on a Bayesian approach. A likelihood including kinematic quantities is used to discriminate the signal from the background. Upper cross section limits are calculated as function of $m_{\tilde{t}}$ and $m_{\tilde{\chi}_{1}^{ \pm}}$, and values from 7 to 12 times higher than the theoretical prediction are computed. As a consequence, no limit on the $\tilde{t}$ mass is derived.

\subsection{The $t \bar{t}$ dilepton final state}

The CDF collaboration searched for $\tilde{t}$ pair production in the final state mimicking the $t \bar{t}$ dilepton signature, using a data sample of $1.9 \mathrm{fb}^{-1}$ [6]. The LSP is assumed to be the lightest neutralino and the $\tilde{t}$ to decay exclusively to a $b$-quark and a $\tilde{\chi}_{1}^{ \pm}$. Considering that $m_{\tilde{\chi}_{1}^{ \pm}}-m_{\tilde{\chi}_{1}^{0}}<$ $m_{W}$, the chargino decay is not limited to a mediation via a $W$ boson, but can also be achieved through additional particles $\left(\tilde{v}, \tilde{\ell}, H^{ \pm}\right)$. The branching fraction of the decay $\tilde{t} \rightarrow b \tilde{\chi}_{1}^{0} \ell v$ is thus enhanced. The analysis is performed in the dielectron, dimuon, and electron-muon channels.

The background mostly originates from $t \bar{t}$ and $Z / \gamma^{*}$ production. The $Z$ boson contribution is vetoed utilizing a variable based on $\mathbb{E}_{T}$. Furthermore, $t \bar{t}$ events are suppressed with a discriminant constructed from the azimuthal separations between the leptons and between the leading jets, and from the sum of the transverse momenta of all the selected objects. Two signal regions are defined: one requires at least one $b$-tagged jet, and the other one consists of events containing anti-tagged jets that are selected with slightly tighter cuts.

The upper cross section limits on the $\tilde{t}$ pair production cross section are set employing the reconstructed $\tilde{t}$ mass as discriminant. To measure the $\tilde{t}$ mass, a method similar to the one used for the $t$-quark in the dilepton channel is adopted, namely the neutrino weighting procedure [7]. The specificity in this analysis is that the $v$ is substituted by the system $\tilde{\chi}_{1}^{0}+v$. The SUSY exclusion region is provided by Figure 4 for the case where $m_{\tilde{\chi}_{1}^{ \pm}}=125.8 \mathrm{GeV}$.

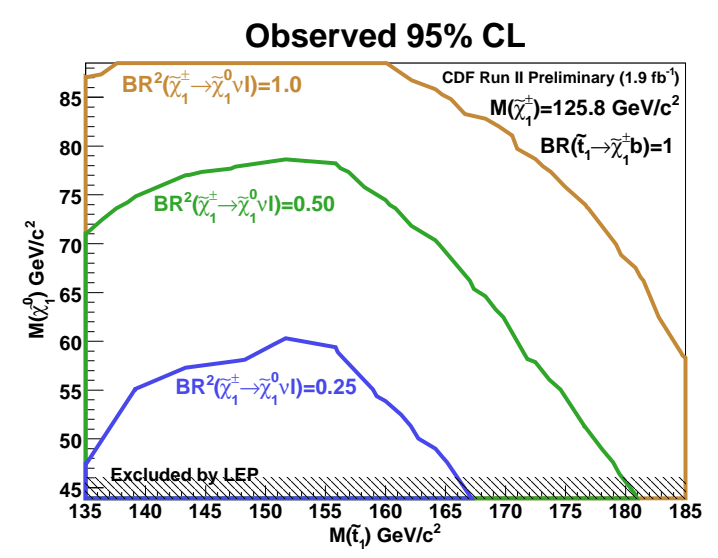

FIGURE 4. Search for $\tilde{t}$ in the $t \bar{t}$ dilepton channel. The mass exclusion region is parametrized as function of $m_{\tilde{\chi}_{1}^{0}}$ and $m_{\tilde{t}}$.

\section{CONCLUSIONS}

Searches for scalar top and bottom quarks have been performed by the D0 and CDF collaborations at the Tevatron. Multiple final states have been investigated using data samples with up to $1.9 \mathrm{fb}^{-1}$. No evidence for such SUSY particles has been observed, but stringent limits on their masses have been derived. In summer 2008, the integrated luminosity collected by both experiments reached nearly $3.5 \mathrm{fb}^{-1}$, and larger luminosities are expected for the full period of Run II.

\section{ACKNOWLEDGMENTS}

We thank the D0 and CDF Physics coordinators and Physics Group conveners for their help.

\section{REFERENCES}

1. S. P. Martin, hep-ph/9709356 (1997).

2. CDF Collaboration, CDF Note 9332 (2008).

3. V. M. Abazov et al., Phys. Lett. B 665, 1 (2008)

4. D0 Collaboration, D0 Note 5598 (2008).

5. D0 Collaboration, D0 Note 5438 (2007).

6. CDF Collaboration, CDF Note 9343 (2008).

7. A. Abulencia et al., Phys. Rev. D 73, 112006 (2006) 